\title{
Simultaneous Analysis of Ursolic Acid and Oleanolic Acid in Guava Leaves Using QuEChERS-Based Extraction Followed by High-Performance Liquid Chromatography
}

\author{
Chang Xu, ${ }^{1}$ Yiyi Liao, ${ }^{1}$ Chunyan Fang, ${ }^{1}$ Makoto Tsunoda, ${ }^{2}$ \\ Yingxia Zhang, ${ }^{1}$ Yanting Song, ${ }^{1}$ and Shiming Deng ${ }^{1}$ \\ ${ }^{1}$ Key Laboratory of Tropical Biological Resources of Ministry of Education and Department of Pharmaceutical Sciences, \\ College of Marine Science, Hainan University, Haikou 570228, China \\ ${ }^{2}$ Graduate School of Pharmaceutical Sciences, The University of Tokyo, 7-3-1 Hongo, Bunkyo-ku, Tokyo 113-0033, Japan \\ Correspondence should be addressed to Yanting Song; songyt@hainu.edu.cn and Shiming Deng; dsm701@126.com
}

Received 3 March 2017; Revised 3 May 2017; Accepted 12 June 2017; Published 11 July 2017

Academic Editor: Angela Chambery

Copyright (C) 2017 Chang Xu et al. This is an open access article distributed under the Creative Commons Attribution License, which permits unrestricted use, distribution, and reproduction in any medium, provided the original work is properly cited.

In this paper, a novel method of QuEChERS-based extraction coupled with high-performance liquid chromatography has been developed for the simultaneous determination of ursolic acid (UA) and oleanolic acid (OA) in guava leaves. The QuEChERSbased extraction parameters, including the amount of added salt, vortex-assisted extraction time, and absorbent amount, and the chromatographic conditions were investigated for the analysis of UA and OA in guava leaves. Under the optimized conditions, the method showed good linearity over a range of $1-320 \mu \mathrm{g} \mathrm{mL} \mathrm{L}^{-1}$, with correlation coefficients above 0.999 . The limits of detection of $\mathrm{UA}$ and $\mathrm{OA}$ were 0.18 and $0.36 \mu \mathrm{g} \mathrm{mL}^{-1}$, respectively. The intraday and interday precision were below 1.95 and $2.55 \%$, respectively. The accuracies of the UA and OA determinations ranged from 97.4 to $111.4 \%$. The contents of UA and OA in the guava leaf samples were 2.50 and $0.73 \mathrm{mg} \mathrm{g}^{-1}$, respectively. These results demonstrate that the developed method is applicable to the simultaneous determination of UA and OA in guava leaves.

\section{Introduction}

Guava (Psidium guajava L.) is a tropical plant that belongs to genus Psidium in the Myrtaceae family. Many parts of this plant, especially its fruits and leaves, are utilized by humans. Its fruits are extremely popular because of their flavour and aroma. Reportedly, guava fruits can also be used to treat diabetes mellitus patients and people who have high level blood cholesterol because the fruits contain abundant amino acids, minerals, and vitamins. Meanwhile, guava leaves have been reported to have pharmacological properties, such as hypoglycaemic [1], antimicrobial [2], anti-inflammatory [3], and antinociceptive [4] activities. Ursolic acid (UA) and oleanolic acid (OA) are isomeric triterpenic acids (Figure 1), which are the principal active components in guava leaves. Pharmacological experiments have demonstrated that UA can modulate a variety of signaling pathways associated with cancer survival and progression and has the ability to cure inflammation or oxidative stress-associated diseases (cancer, cardiovascular disease, and neurodegeneration) [5], and OA displays several pharmacological activities, such as antiinflammatory, antioxidant, anticancer, and hepatoprotective effects [6]. The contents of UA and OA are very low, and their analysis was easily interfered by the complex matrix compounds in guava leaves. Therefore, the establishment of an accurate and easy analytical method for these compounds is particularly important.

For the trace analysis of target analytes, sample preparation, which includes both clean-up and preconcentration before chromatographic analysis, is indispensable for many types of samples. Several sample pretreatment methods such as ultrasound-assisted extraction (UAE) [7], microwaveassisted extraction (MAE) [8], accelerated solvent extraction (ASE) [9], solvent bar microextraction (SBME) [10], and solid-phase extraction (SPE) [11] have been reported for the extraction and enrichment of UA and OA from different 


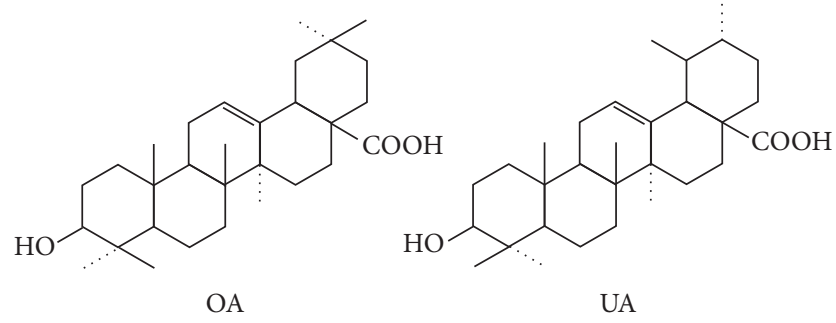

FIgURE 1: Chemical structures of oleanolic acid (OA) and ursolic acid (UA).

matrices prior to analysis by gas chromatography (GC) or liquid chromatography (LC). Among these methods, SPE is increasingly being used as a sample preparation technique because of its advantages of reducing sample handling, labour, and organic solvent consumption. The most popular sorbents for SPE are octadecyl $\left(\mathrm{C}_{18}\right)$-bonded silica or polymeric sorbents. Other sorbents that enhance selectivity are immunosorbents and molecularly imprinted polymers (MIPs). However, the SPE method often fails to remove interferences when a large number of samples is extracted. Other problems include low recovery, incomplete elution, poor conditioning, and high variability due to method and operator variability. However, the main disadvantage of SPE is its unconfirmed effectiveness at extracting water-soluble metabolites.

Currently, a new sample preparation method, QuEChERS, which stands for "quick, easy, cheap, effective, rugged, and safe," combined with GC or LC, has been used for the multiclass, multiresidue analysis of pesticides and natural products in fruits, vegetables, and herbs. QuEChERS can serve as a template for modification because of its characteristic flexibility and can be adjusted for various matrices depending on the analyte properties, matrix composition, equipment, and analytical technique. Oshita and Jardim compared QuEChERS-based dispersive and cartridge SPE for the analysis of pesticides in strawberries and found that the QuEChERS-based dispersive SPE extraction clean-up of the strawberry extracts was more efficient, achieving higher pesticide recoveries and better clean-up via interference removal than those reported to date [12]. Tölgyessy et al. have successfully applied the QuEChERS method with Dual dispersive SPE and gas chromatography to the determination of ten chlorinated priority substances in fish [13]. Li et al. proposed an effective method based on QuEChERS to extract triazines and phenylureas from milk and yogurt [14]. Raihanah et al. used the QuEChERS preparation method to analysis imidacloprid residue in paddy samples [15].

The aim of this work was to develop an effective method based on QuEChERS-based extraction coupled with highperformance LC (HPLC) for the simultaneous detection of $\mathrm{UA}$ and $\mathrm{OA}$ in guava leaves. In this study, QuEChERS Kits were used for the pretreatment of guava leaves. The method utilizes a combination of primary and secondary amines (PSA) to remove organic acids, anhydrous $\mathrm{MgSO}_{4}$ to reduce the remaining water, and $\mathrm{C}_{18}$ to remove lipids and waxes. Besides, the extraction parameters affecting the sample recoveries were optimized, and the obtained accuracy and precision demonstrated that the proposed method is applicable for the determination of $\mathrm{UA}$ and $\mathrm{OA}$ in guava leaves.

\section{Materials and Methods}

2.1. Materials. Standards of UA and OA (purity is more than 98\%) were purchased from Shanghai Yuanye Biotechnology Co., Ltd. (Shanghai, China). Triethylamine and acetate (HPLC grade) were obtained from Aladdin Industrial Corporation (Shanghai, China). Methanol (HPLC grade) was purchased from Fisher Scientific (Beijing, China). Ultrapure water was obtained from a Milli-Q water purification system (Millipore, USA). All the other reagents were analytical grade. Extraction was performed using Agilent SampliQ QuEChERS AOAC Extraction Kits ( $\mathrm{p} / \mathrm{n}$ 5982-5122), Agilent Bond Elut Florisil (12256014), Dikma ProElut $\mathrm{C}_{18}(500 \mathrm{mg} / 6 \mathrm{~mL})$, and Dikma ProElut PLS (500 mg/6 mL).

2.2. Standard Solutions. Stock solutions of UA and OA (1 $\mathrm{mg} \mathrm{mL} \mathrm{m}^{-1}$ ) were prepared in methanol. Mixed working solutions, containing $0.4 \mathrm{mg} \mathrm{mL}^{-1} \mathrm{UA}$ and $0.2 \mathrm{mg} \mathrm{mL}^{-1} \mathrm{OA}$, were prepared by appropriate dilution of the stock solution with methanol. All solutions were stored at $4^{\circ} \mathrm{C}$ before use.

2.3. Sample Preparation. Guava leaves were collected from the campus of Hainan University and pulverized into powder. Approximately $2.5 \mathrm{~g}$ of the powder was accurately weighed, transferred into a clear beaker, dissolved in $15 \mathrm{~mL}$ of metha$\mathrm{nol}$, and then ultrasounded for $1 \mathrm{~h}$. The solution was transferred to a $25 \mathrm{~mL}$ volumetric flask, brought to volume, and filtered through a funnel. The filtrate was subsequently centrifuged (Hermle, Germany) at $13000 \mathrm{rpm}$ for $5 \mathrm{~min}$ at $25^{\circ} \mathrm{C}$. Finally, the supernatant was collected.

2.4. Procedure. The extraction procedure consisted of several steps. First, the centrifuged sample $(2 \mathrm{~mL})$ containing the analytes was transferred to a QuEChERS Kit containing $150 \mathrm{mg}$ of $\mathrm{MgSO}_{4}, 50 \mathrm{mg}$ of PSA, and $50 \mathrm{mg}$ of $\mathrm{C}_{18} \mathrm{EC}$ sorbent. After adjusting the $\mathrm{pH}$ to 7 by adding appropriate amounts of $10 \mathrm{~mol} / \mathrm{L} \mathrm{NaOH}$ solutions, the capped tubes were shaken for $3 \mathrm{~min}$ by hand and then vortexed for $1 \mathrm{~min}$ on a mechanical shaker to facilitate the dispersion of the sample with $3 \% w / v$ salt addition. Finally, the capped tubes were centrifuged at $8000 \mathrm{rpm}$ for $5 \mathrm{~min}$ at $4^{\circ} \mathrm{C}$. The supernatant was filtered and then evaporated until near dryness with gentle nitrogen stream at $40^{\circ} \mathrm{C}$. Finally, the residue was dissolved in $2 \mathrm{~mL}$ methanol, filtered through a $0.2 \mu \mathrm{m}$ nylon syringe filter (JinTeng, China), and $5 \mu \mathrm{L}$ of filtrate was injected into the HPLC.

Agilent Bond Elut Florisil column was activated with acetone/n-hexane $(5 \mathrm{~mL}, 1: 9, \mathrm{v} / \mathrm{v})$ and conditioned with $\mathrm{n}$ hexane $(5 \mathrm{~mL})$ before application; after the sample had passed through the column, $2 \mathrm{~mL}$ of the methanol extractants was loaded on the cartridge and then eluted with acetone/nhexane $(5 \mathrm{~mL}, 1: 9, \mathrm{v} / \mathrm{v})$. The eluent was collected and then evaporated until near dryness with gentle nitrogen stream at $40^{\circ} \mathrm{C}$ [16]. Finally, the residue was dissolved in $2 \mathrm{~mL}$ methanol, filtered through a $0.2 \mu \mathrm{m}$ nylon syringe filter (JinTeng, China), and $5 \mu \mathrm{L}$ of filtrate was injected into the HPLC. 


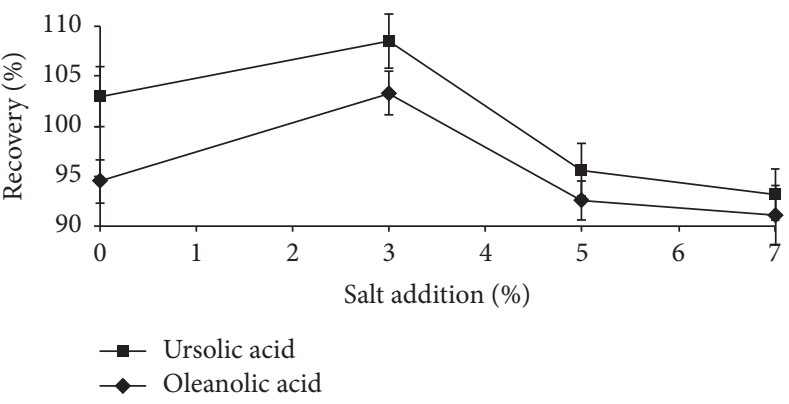

(a)

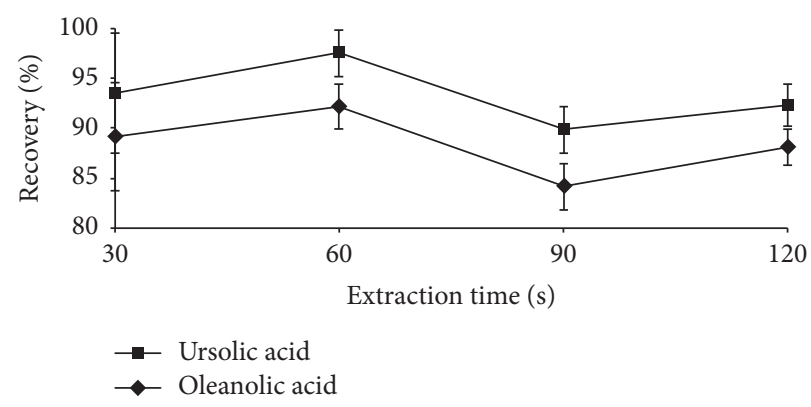

(b)

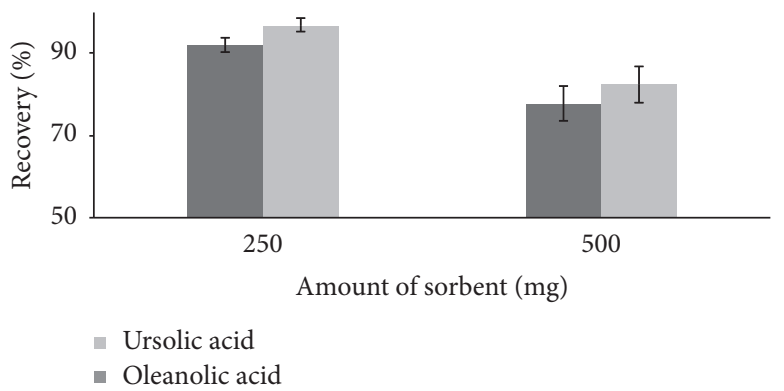

(c)

FIGURE 2: Effect of salt addition (a), extraction time (b), and sorbent amount (c) on the QuEChERS-based extraction of standard solutions.

Dikma ProElut $\mathrm{C}_{18}$ and Dikma ProElut PLS column were activated with $5 \mathrm{~mL}$ of methanol and $5 \mathrm{~mL}$ of Milli-Q water before use, respectively. Two microlitres of the methanol extracts passed through the column and then were washed with $5 \mathrm{~mL}$ of Milli-Q water and $5 \mathrm{~mL}$ of phosphate buffer $(0.05 \mathrm{M}, \mathrm{pH}=8.5)$, the eluent discarded. The analyte was then eluted with $5 \mathrm{~mL}$ of methanol; the eluent was collected and then evaporated until near dryness with gentle nitrogen stream at $40^{\circ} \mathrm{C}$ [17]. Finally, the residue was dissolved in $2 \mathrm{~mL}$ methanol, filtered through a $0.2 \mu \mathrm{m}$ nylon syringe filter (JinTeng, China), and $5 \mu \mathrm{L}$ of filtrate was injected into the HPLC.

2.5. Chromatographic Conditions. HPLC analysis of UA and OA was performed on an HPLC instrument as described above. The mobile phase consisted of methanol-wateracetate-triethylamine $(90: 10: 0.04: 0.02, \mathrm{v} / \mathrm{v} / \mathrm{v} / \mathrm{v})$. The flow rate was $1.0 \mathrm{~mL} \mathrm{~min}^{-1}$, with $\mathrm{UV}$ detection at $210 \mathrm{~nm}$. The column temperature was maintained at $30^{\circ} \mathrm{C}$. Five microlitres of the centrifuged supernatant was injected into an HPLC system equipped with a Waters 1525 pump and 2489 detector (Milford, USA) and a Silversil $\mathrm{C}_{18}$ analytical column $(250 \mathrm{~mm}$ $\times 4.6 \mathrm{~mm}$ ID, $5 \mu \mathrm{m}$ ) from Dikma Technologies Inc. (Beijing, China). These were performed based on previous report [18].

2.6. Optimization of QuEChERS Procedure. The QuEChERSbased extraction parameters, including the added salt amount, extraction time, and absorbent amount, were investigated. The experiments were all carried out in triplicate using the standard solutions. The results were then checked in guava leaf extract.
2.7. Validation Study. A series of standard solutions containing UA and OA $\left(1,10,20,40,80,160\right.$, and $\left.320 \mu \mathrm{gL}^{-1}\right)$ were prepared for the establishment of the calibration curve. The intraday precision was determined by analyzing the QuEChERS-based extracted guava leave samples five times in the same day. The interday precision experiment was performed by analyzing the QuEChERS-based extracted guava leave samples once a day for five consecutive days. The reproducibility was evaluated by six QuEChERS-based extracted samples in the completely same procedure. The recovery of the method was assessed by the addition of standard solutions with different concentrations into the extracted sample obtained in Section 2.3. The recovery $(R)$ was calculated based on the following equation:

$$
\begin{aligned}
R= & \frac{[\text { analyte }]_{\text {sample with spike }}-[\text { analyte }]_{\text {sample without spike }}}{[\text { analyte }]_{\text {added }}} \\
& \times 100 \%
\end{aligned}
$$

\section{Results and Discussion}

3.1. Effect of the Amount of Salt Addition. Salt was added directly to partition the organic and aqueous layers through a higher salting-out effect. A concentration of $0-7 \%(w / v)$ sodium chloride was added to the standard solution to examine the effect of the amount of salt addition on the extraction efficiency. The peak areas of the analytes increased when the $\mathrm{NaCl}$ concentration was increased from $0-3 \%(w / v)$ and decreased when the $\mathrm{NaCl}$ concentration was further increased (Figure 2(a)). The addition of salt into solution 
can significantly reduce the solubility of polar substances; however, when the salt concentration is too high, the viscosity of the solution will increase, resulting in reduced adsorbent extraction capacity. Therefore, 3\% $(w / v)$ salt addition was utilized in further studies.

3.2. Effect of the Extraction Time. The extraction time is particularly important for the mass transfer process, and the vortex extraction time was varied from 30 to $120 \mathrm{~s}$. The extraction efficiency increased when the extraction time was extended from 30 to $60 \mathrm{~s}$ and slightly decreased when the extraction time was extended to $120 \mathrm{~s}$ (Figure 2(b)). This decrease may have been due to the back extraction of analytes from the sorbent into the sample solution. Therefore, $60 \mathrm{~s}$ was used in subsequent analyses as the optimum extraction time.

3.3. Effect of the Sorbent Amount. The effect of the sorbent amount on the extraction efficiency was investigated at $250 \mathrm{mg}$ and $500 \mathrm{mg}$ of sorbent. A total of $250 \mathrm{mg}$ of sorbent consisted of $150 \mathrm{mg}$ of $\mathrm{MgSO}_{4}, 50 \mathrm{mg}$ of PSA, and $50 \mathrm{mg}$ of $\mathrm{C}_{18} \mathrm{EC}$, and $500 \mathrm{mg}$ of sorbent contained the same components in double the quantity. The results presented in Figure 2(c) indicate that the maximum recovery was achieved by using $250 \mathrm{mg}$ of sorbent to extract $2 \mathrm{~mL}$ of sample solution. Hence, $250 \mathrm{mg}$ of sorbent was used in the subsequent experiments.

3.4. Optimum QuEChERS-Based Extraction Conditions of Guava Leaf Samples. Additional experiments were performed to check the optimum QuEChERS-based extraction conditions of guava leaf samples. The results showed that the optimum QuEChERS-based extraction conditions are consistent with what we did in standard solutions with the presence of other matrix analytes.

3.5. Method Validation. Under the optimum conditions, the proposed method was validated in terms of the linearity, limit of detection (LOD), limit of quantification (LOQ), precision, and recovery.

3.5.1. Linearity, Limit of Detection, and Limit of Quantification. Good linearity was observed for UA and OA, with correlation coefficients $r^{2}$ of 0.9997 and 0.9991 , respectively. The LODs and LOQs, based on signal-to-noise ratios of $3(S / N=3)$ and $10(S / N=10)$, were found to be in the range of $0.18-0.36$ and $0.6-1.2 \mu \mathrm{g} \mathrm{mL}^{-1}$, respectively. These results illustrate the lower detection limits of UA and OA of the proposed method. All of the analytical characteristics are summarized in Table 1.

3.5.2. Intraday and Interday Precision. To evaluate the precision of the method, five similar experiments were carried out with samples on the same day and on five consecutive days. The intraday relative standard deviation (RSD\%) ranged from 1.48 to $1.95 \%$ and the interday precision ranged from 1.18 to $2.55 \%$ (Table 1 ). The relative standard deviation (RSD) for the peak area is $2.52 \%$. These results show the good reproducibility and precision of this method.
3.5.3. Recovery. A recovery study was carried out by spiking methanol extracts in Section 2.3 with three different concentrations of UA $\left(60,120\right.$, and $\left.180 \mu \mathrm{g} \mathrm{mL}^{-1}\right)$ and $\mathrm{OA}(12,24$, and $\left.36 \mu \mathrm{g} \mathrm{mL}^{-1}\right)$. The results summarized in Table 1 show that the recoveries of UA and OA were within a range of 97.4 to $111.4 \%$, and, in RSD, they ranged from 0.38 to $7.14 \%$. UA and OA are planar molecules and QuEChERS sorbents are not efficient to retain planar molecules [19]; therefore, good recoveries were achieved.

3.6. Analysis of Guava Leaf Samples. The developed method was applied to the determination of UA and OA in guava leaf samples. The contents of UA and OA in the guava leaf samples were 2.50 and $0.73 \mathrm{mg} \mathrm{g}^{-1}$, respectively. These values were in good agreement with those reported in previous study [20]. Figure 3 shows HPLC chromatograms of standard solutions of UA and OA (a), a blank guava leaf sample (b), and a guava leaf sample after QuEChERS-based extraction (c).

3.7. Comparison with Other Reported Methods. QuEChERS method was compared with other three solid-phase columns, including Agilent Bond Elut Florisil column, Dikma ProElut $\mathrm{C}_{18}$ column, and Dikma ProElut PLS column. Figure 4 presented that the peak areas of UA and OA obtained by QuEChERS method were obviously higher than the other three columns and indicated that QuEChERS method achieved higher recovery of UA and OA in guava leaves comparing with other three extraction methods. Moreover, the proposed method yields higher recoveries and reproducible results. Overall, QuEChERS-based extraction combined with HPLC$\mathrm{UV}$ is a useful alternative approach for the preconcentration and determination of UA and OA in complex matrices.

\section{Conclusions}

In this study, a method consisting of QuEChERS-based extraction combined with HPLC was developed and optimized to quantitatively determine trace levels of UA and $\mathrm{OA}$ in guava leaves. The extraction and clean-up procedures are simple and time-saving. Moreover, compared with other solid-phase extraction methods, the proposed method yields higher recoveries of UA and OA in guava leaves. The validated method provides good precision, a wide linear range, and low LOD and LOQ in the $\mu \mathrm{g} \mathrm{mL}^{-1}$ range for the analysis of guava leaf samples. The results suggest that QuEChERSbased extraction combined with HPLC-UV can be a useful alternative approach for the preconcentration and determination of UA and OA in complex matrices.

\section{Conflicts of Interest}

The authors declare that there are no conflicts of interest regarding the publication of this paper.

\section{Acknowledgments}

The work was supported by the Natural Science Foundation of Hainan Province [20158363]; the National Natural Science 


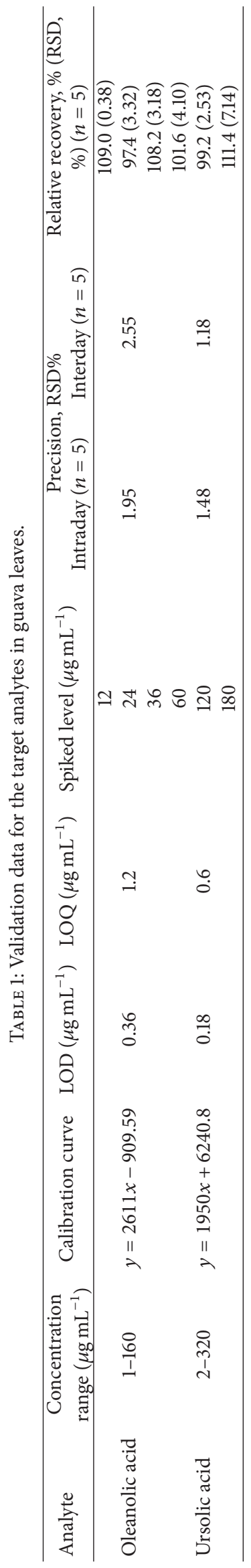




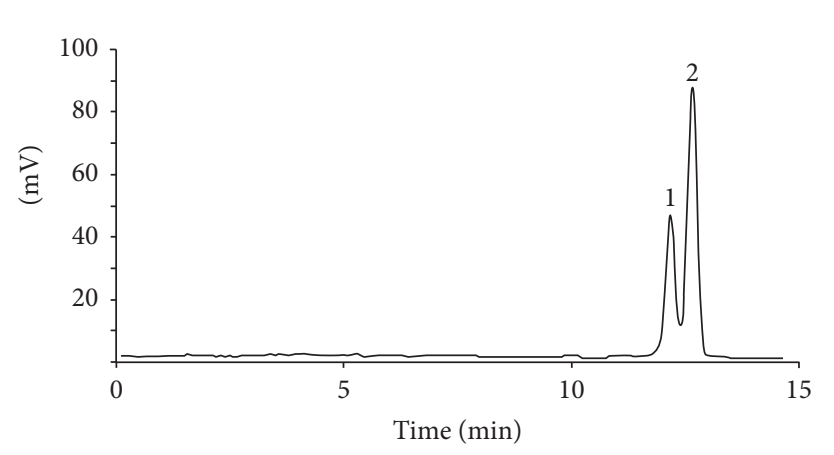

(a)

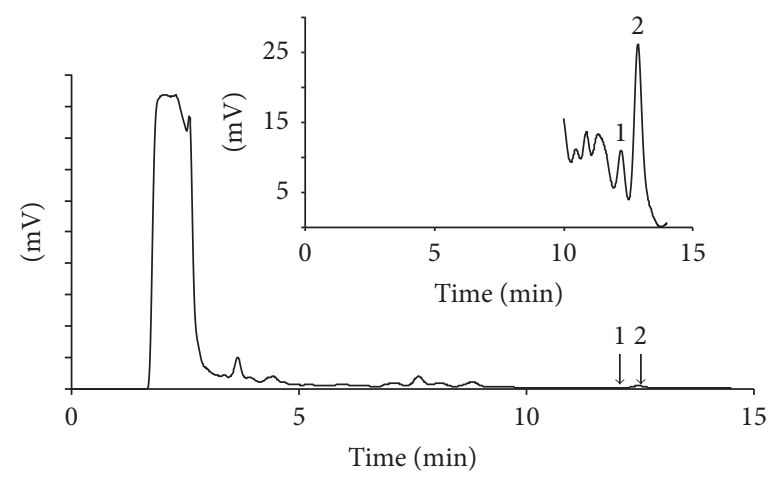

(b)

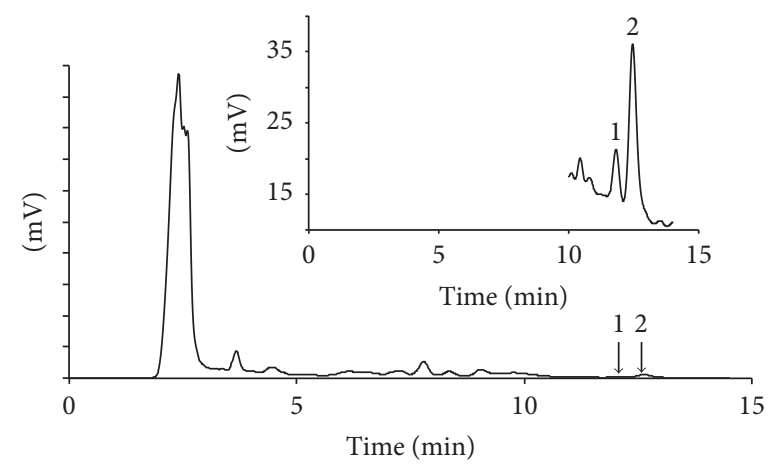

(c)

FIGURE 3: HPLC chromatograms of standard solutions of UA and OA (a), a blank guava leaf sample (b), and a guava leaf sample after QuEChERS-based extraction (c). The embedded graphs are the magnified view of the chromatograms. Peaks in the chromatogram: (1) OA, (2) UA.

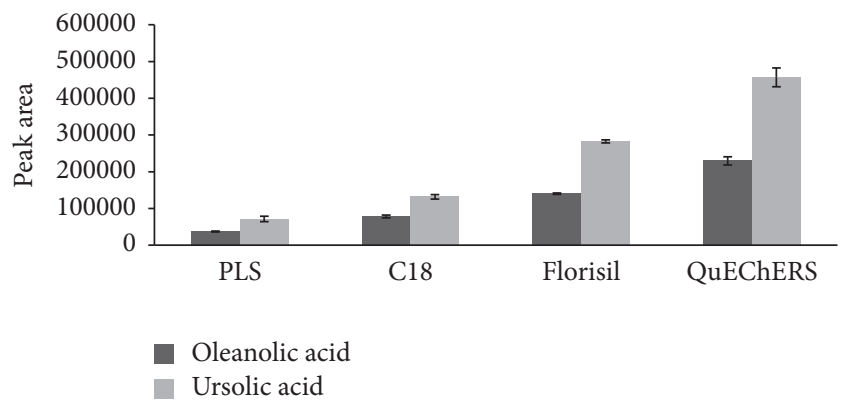

FIgURE 4: Peak area of the guava leave samples in different extraction columns.

Foundation of China [21505029]; and the Scientific Research Foundation for the Returned Overseas Chinese Scholars, State Education Ministry. The authors also offer their sincere gratitude to Kobayashi International Scholarship Foundation.

\section{References}

[1] C.-W. Liu, Y.-C. Wang, C.-C. Hsieh, H.-C. Lu, and W.-D. Chiang, "Guava (Psidium guajava Linn.) leaf extract promotes glucose uptake and glycogen accumulation by modulating the insulin signaling pathway in high-glucose-induced insulinresistant mouse FL83B cells," Process Biochemistry, vol. 50, no. 7, pp. 1128-1135, 2015.

[2] A. A. De Araújo, L. A. Lira Soares, M. R. Assunção Ferreira et al., "Quantification of polyphenols and evaluation of antimicrobial, analgesic and anti-inflammatory activities of aqueous and acetone-water extracts of Libidibia ferrea, Parapiptadenia rigida and Psidium guajava," Journal of Ethnopharmacology, vol. 156, pp. 88-96, 2014.

[3] M.-H. Kim, J. N. Kim, S. N. Han, and H.-K. Kim, "Ursolic acid isolated from guava leaves inhibits inflammatory mediators and reactive oxygen species in LPS-stimulated macrophages," Immunopharmacology and Immunotoxicology, vol. 37, no. 3, pp. 228-235, 2015.

[4] R. M. Pérez Gutiérrez, S. Mitchell, and R. Vargas Solis, "Psidium guajava: a review of its traditional uses, phytochemistry and pharmacology," Journal of Ethnopharmacology, vol. 117, no. 1, pp. $1-27,2008$.

[5] D. Kashyap, H. S. Tuli, and A. K. Sharma, "Ursolic acid (UA): A metabolite with promising therapeutic potential," Life Sciences, vol. 146, pp. 201-213, 2016.

[6] T. S. Melo, C. R. Gattass, D. C. Soares et al., "Oleanolic acid (OA) as an antileishmanial agent: Biological evaluation and in silico mechanistic insights," Parasitology International, vol. 65, no. 3, pp. 227-237, 2016.

[7] E.-Q. Xia, Y.-Y. Yu, X.-R. Xu, G.-F. Deng, Y.-J. Guo, and H.-B. $\mathrm{Li}$, "Ultrasound-assisted extraction of oleanolic acid and ursolic 
acid from Ligustrum lucidum Ait," Ultrasonics Sonochemistry, vol. 19, no. 4, pp. 772-776, 2012.

[8] E.-Q. Xia, B.-W. Wang, X.-R. Xu, L. Zhu, Y. Song, and H.B. Li, "Microwave-assisted extraction of oleanolic acid and ursolic acid from Ligustrum lucidum ait," International Journal of Molecular Sciences, vol. 12, no. 8, pp. 5319-5329, 2011.

[9] M. Wójciak-Kosior, I. Sowa, R. Kocjan, and R. Nowak, "Effect of different extraction techniques on quantification of oleanolic and ursolic acid in Lamii albi flos," Industrial Crops and Products, vol. 44, pp. 373-377, 2013.

[10] Y. Hao, X. Chen, S. Hu, and X. Bai, "Solvent bar microextraction with HPLC for determination and protein-binding characteristics of oleanolic acid and ursolic acid," Chromatographia, vol. 77, no. 3-4, pp. 359-363, 2014.

[11] M. Tarvainen, J.-P. Suomela, H. Kallio, and B. Yang, “Triterpene acids in plantago major: Identification, quantification and comparison of different extraction methods," Chromatographia, vol. 71, no. 3-4, pp. 279-284, 2010.

[12] D. Oshita and I. C. S. F. Jardim, "Evaluation of dispersive and cartridge SPE clean-up procedures using the modified QuEChERS method for the analysis of pesticides in strawberries," Analytical Methods, vol. 7, no. 3, pp. 982-989, 2015.

[13] P. Tölgyessy, Z. Miháliková, and M. Matulová, “Determination of selected chlorinated priority substances in fish using quechers method with dual dspe clean-up and gas chromatography," Chromatographia, vol. 79, no. 21-22, pp. 1561-1568, 2016.

[14] N. Li, L. Lei, L. Nian et al., "A modified QuEChERS method for the determination of some herbicides in yogurt and milk by high performance liquid chromatography," Talanta, vol. 105, pp. 219-228, 2013.

[15] C. Raihanah, H. Zailina, Y. B. Ho, M. E. Saliza, and M. Norida, "Ultra high performance liquid chromatography technique to determine imidacloprid residue in rice using QuEChERS method," International Food Research Journal, vol. 23, no. 4, pp. 1396-1402, 2016.

[16] L. Shuying, W. Yihu, S. Qianqian, G. Wenjun, and Z. Guonian, "Residue determination of plant activator benzo-1,2,3thiadiazole-7- carboxylic acid 2-benzoyloxyethyl ester in water, soil, and vegetable by gas chromatography with tandem mass spectrometry," Journal of Separation Science, vol. 38, no. 11, pp. 1900-1906, 2015.

[17] C. Yan, B. Zhang, W. Liu, F. Feng, Y. Zhao, and H. Du, "Rapid determination of sixteen sulfonylurea herbicides in surface water by solid phase extraction cleanup and ultrahigh-pressure liquid chromatography coupled with tandem mass spectrometry," Journal of Chromatography B: Analytical Technologies in the Biomedical and Life Sciences, vol. 879, no. 30, pp. 3484-3489, 2011.

[18] H. Wang, Y. Wu, and Y. Huang, "Simultaneous Determination of Oleanolic Acid and Ursolic Acid in Bai huasheshecao by HPLC-DAD," Pharmacy and Clinics of Chinese Materia Medica, vol. 7, no. 1, pp. 20-22, 2016.

[19] E. Dankyi, D. Carboo, C. Gordon, and I. S. Fomsgaard, "Application of the QuEChERS procedure and LC-MS/MS for the assessment of neonicotinoid insecticide residues in cocoa beans and shells," Journal of Food Composition and Analysis, vol. 44, pp. 149-157, 2015.

[20] J. L. Huang and Z. X. Zhang, "The simultaneous determination of oleanolic and ursolic acid in guava leaves by gas chromatography-mass spectrometry," Analysis and Testing Technology and Instruments, vol. 3, pp. 178-181, 2005. 

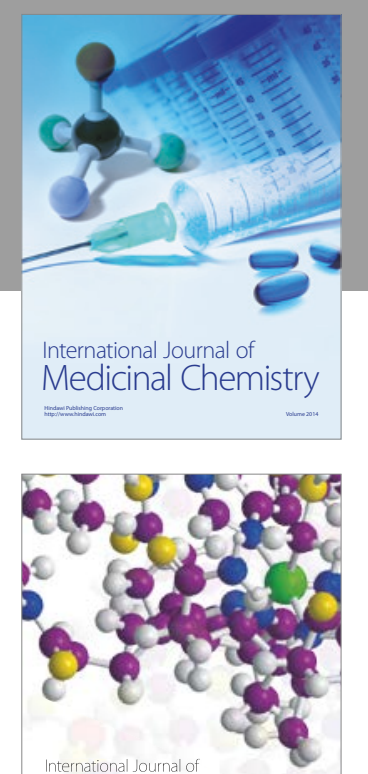

Carbohydrate Chemistry

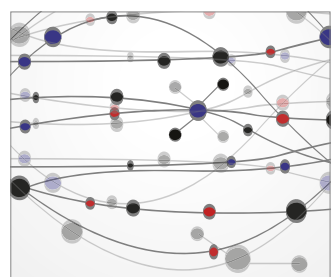

The Scientific World Journal
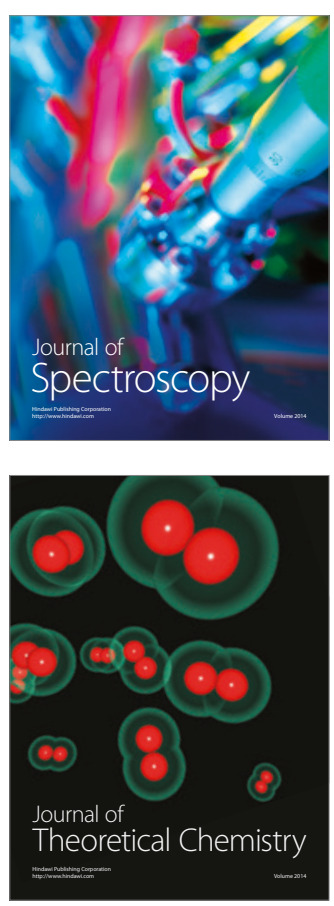
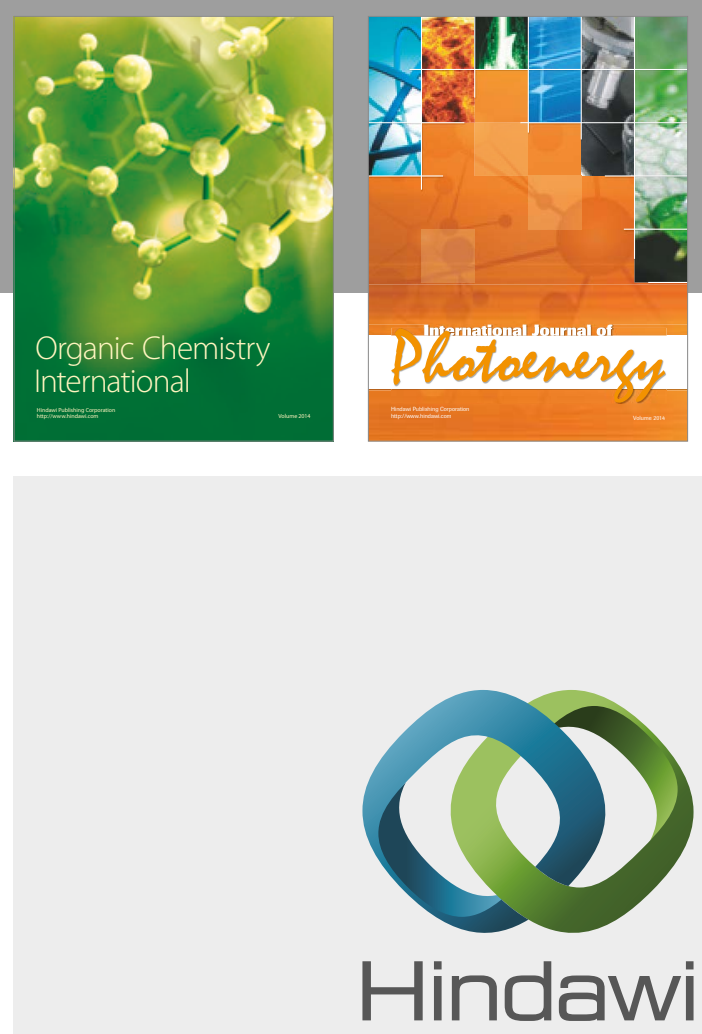

Submit your manuscripts at

https://www.hindawi.com

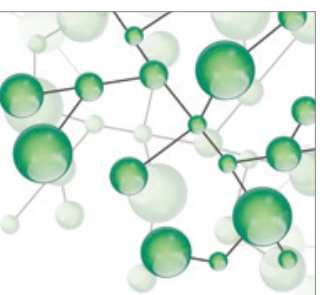

International Journal of

Inorganic Chemistry

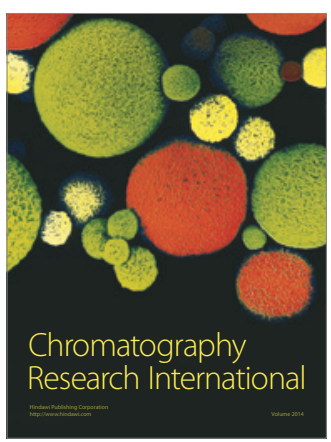

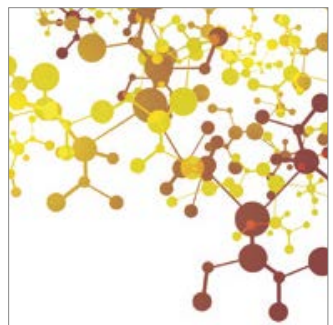

Applied Chemistry
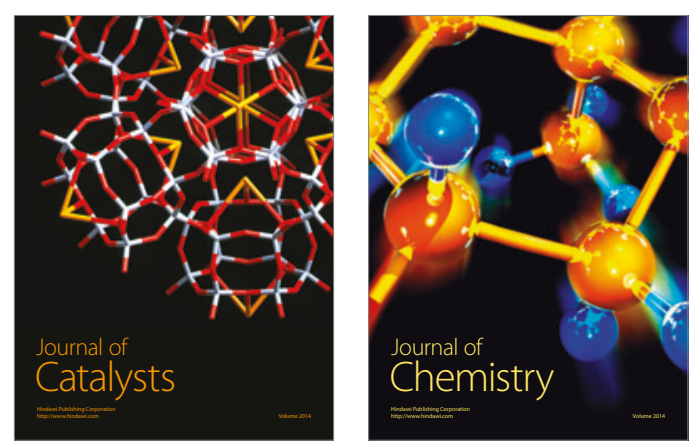
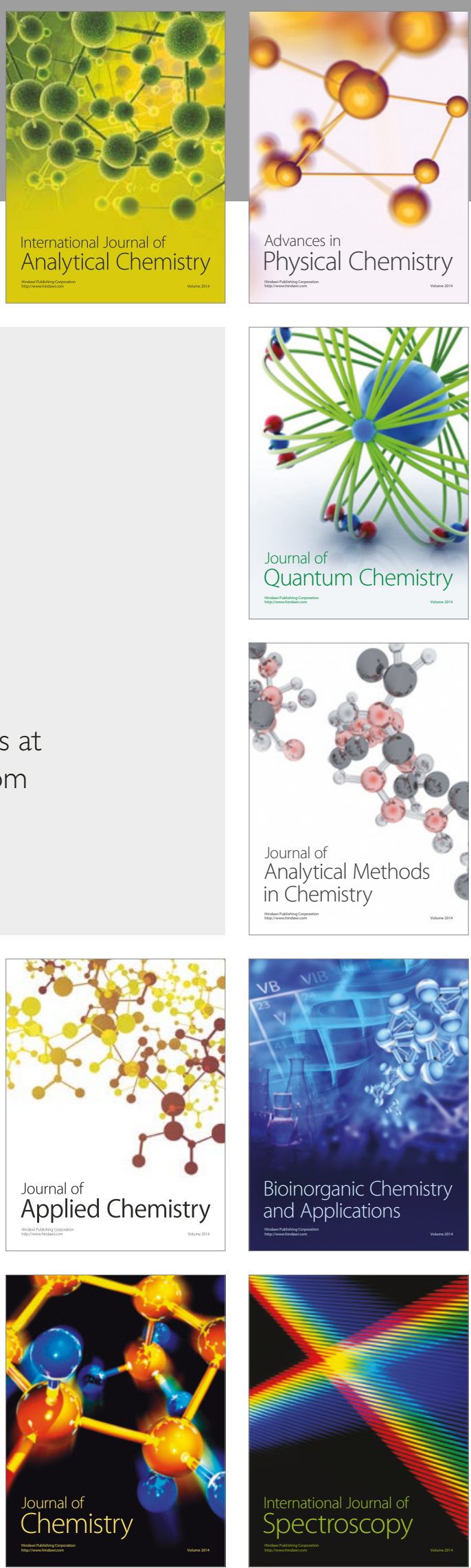